\title{
Mineral Chemistry of Wehrlite Xenoliths Hosted in Basalts from the SW of Hosséré Dammougalré (Adamawa Plateau, Cameroon): Thermobarometric Implications
}

\author{
Isaac Bertrand Gbambié Mbowou1 ${ }^{*}$, Dagwaï Nguihdama², Fadimatou Ngounouno Yamgouot ${ }^{3}$, \\ Mama Ntoumbe1, Abdel Aziz Youpoungam', Ismaïla Ngounouno1
}

${ }^{1}$ School of Geology and Mining Engineering, University of Ngaoundere, Ngaoundere, Cameroon

${ }^{2}$ Department of Life and Earth Sciences, Higher Teachers' Training College, University of Maroua, Maroua, Cameroun

${ }^{3}$ Department of Earth Sciences, Faculty of Science, University of Ngaoundere, Ngaoundere, Cameroon

Email: *mbowou2000@yahoo.fr

How to cite this paper: Mbowou, I.B.G., Nguihdama, D., Yamgouot, F.N., Ntoumbe, M., Youpoungam, A.A. and Ngounouno, I. (2017) Mineral Chemistry of Wehrlite Xenoliths Hosted in Basalts from the SW of Hosséré Dammougalré (Adamawa Plateau, Cameroon): Thermobarometric Implications. Open Journal of Geology, 7, 1465-1477. https://doi.org/10.4236/ojg.2017.710098

Received: August 24, 2017

Accepted: October 13, 2017

Published: October 16, 2017

Copyright $\odot 2017$ by authors and Scientific Research Publishing Inc. This work is licensed under the Creative Commons Attribution International License (CC BY 4.0).

http://creativecommons.org/licenses/by/4.0/

\begin{abstract}
Wehrlite samples (size: $\sim 4 \mathrm{~cm}$ ) hosted in basaltic lavas from the SW of Hosséré Dammougalré are located in the western Adamawa Plateau. Porphyritic and allotriomorphic texture characterize respectively host Basalt and wehrlite xenoliths. The phenocrysts of olivine $\left(\mathrm{Fo}_{68-74}\right)$, and Ti-magnetite are scattered in host basalt. Wehrlite xenoliths $(\sim 4 \mathrm{~cm}$ size) contain $\mathrm{Cr}$-rich clinopyroxene (diopise-augite), olivine $\left(\mathrm{Fo}_{76-88}\right)$ and chromiferous spinel. Equilibrium temperatures calculated from $\mathrm{Fe} / \mathrm{Mg}$ exchange reaction for olivine/spinel vary between $944^{\circ} \mathrm{C}$ and $1102^{\circ} \mathrm{C}$. The wehrlite olivine crystals with low Fo $(<90)$ indicate a re-equilibration of $\mathrm{Fe}-\mathrm{Mg}$ in the host basalt at low temperatures. All the analyzed wehrlite clinopyroxenes have crystallized at high pressures as evidenced by the $\mathrm{Al}^{\mathrm{vi}}$ and $\mathrm{Al}^{\mathrm{liv}}$ contents. The studied spinelbearing wehrlite xenoliths represent probably the residual portions of the upper mantle, which are an important source of information about lithospheric composition and thermal evolution beneath the Adamawa Plateau.
\end{abstract}

\section{Keywords}

Wehrlite, Adamawa Plateau, Hosséré Dammougalré, Mineral Chemistry, Geothermometry

\section{Introduction}

The accidental fragments called rock xenoliths, piked up by the turbulent host 
magma on its ascent, allowed to characterize petrologically the Earth's upper mantle. They are an important source of information about lithospheric composition and thermal evolution in mantle regions associated with alkaline volcanism [1]. In the Adamawa Plateau (Figure 1), previously ultramafic xenoliths data are from Dibi area ([2] [3]), Youkou maar [4], Ngao Voglar [5] and Hosséré Garba [6]. These ultramafic xenoliths studied are chiefly of dunite and lherzolite composition. Thus, the discovery of the wehrlite samples from the SW of Hosséré Dammougalré could improve the knowledge of the upper mantle beneath the Adamawa Plateau. Indeed, wehrlite samples hosted in basaltic lavas from the SW of Hosséré Dammougalré are located in the western Adamawa Plateau (Figure 1). Adamawa Plateau is a tectonomagmatic domain bordered by faults oriented $\mathrm{N} 70^{\circ} \mathrm{E}$ [7]. This plateau presented as the horst, is situated to the north of the Congo Craton, as the result of the fold belt shearing, oriented WSW-ENE [8].

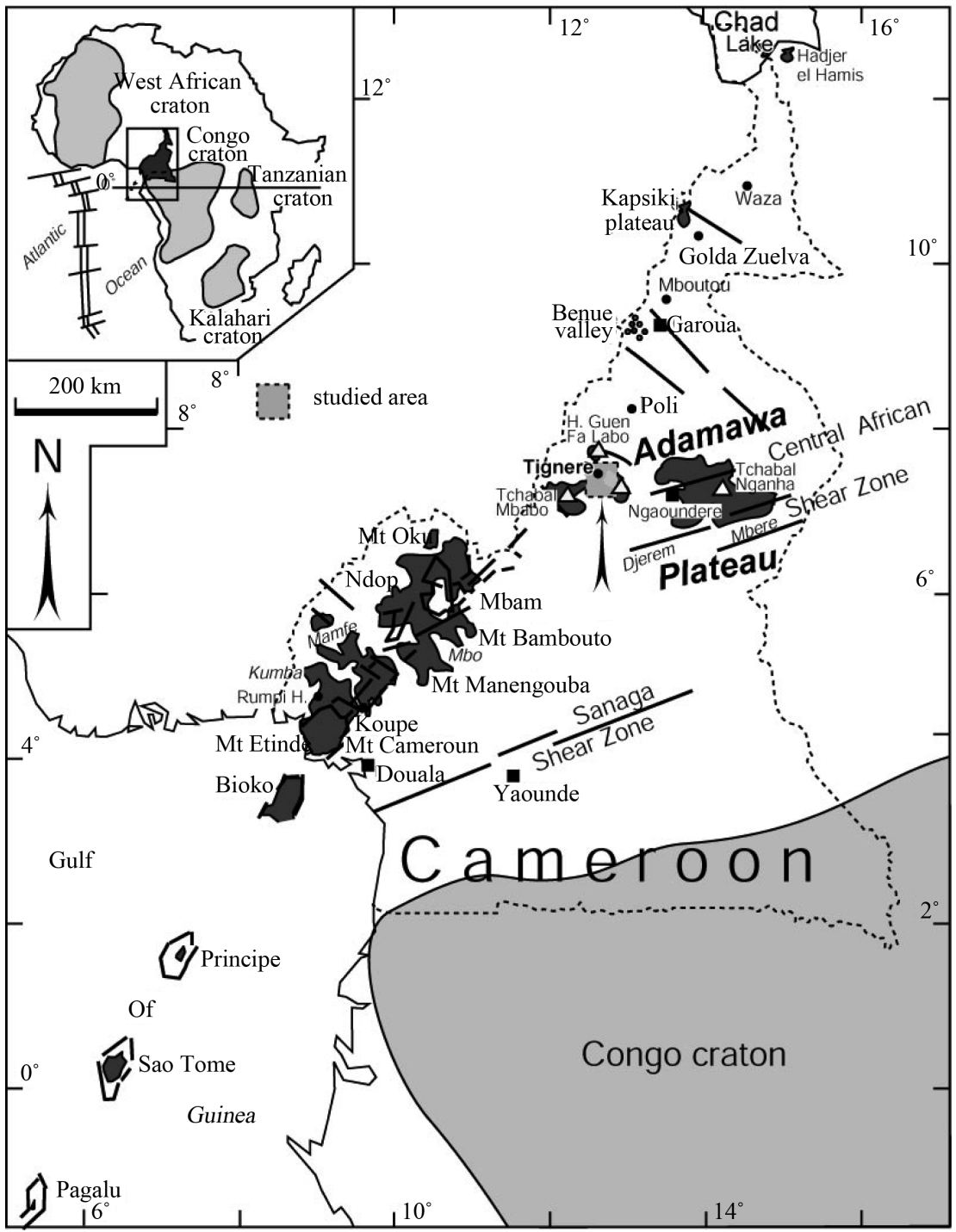

Figure 1. Location of the studied zone in the Adamawa Plateau domain. 
The aims of this paper are to present the first petrographical, mineral chemistry and thermobarometric data of the wehrlite xenoliths from the SW of Hosséré Dammougalre, chiefly to enhance the knowledge of upper mantle beneath the Adamawa Plateau.

\section{Geological Description of the Studied Zone}

The geological sketch established for the studied zone is presented (Figure 2). The trachytic outcrops of Hosséré Dammougalré and Hosséré Doro are surrounded by several basaltic units (lower, middle and upper). The lower basaltic units were almost completely weathered and converted into black reddish ferralitic soils or the clusters of residual ferruginous vacuolar cuirasses. The intermediate or middle basaltic units were fragmented into angular or rounded blocks of various sizes $(5-50 \mathrm{~cm})$, slightly weathered and dispersed. The upper basaltic units were represented by the blocks accumulated as small isolated hills (diameter: $\sim 500 \mathrm{~m}$; height $<30 \mathrm{~m})$. These hills consist of small prismatic blocks $(0.2$ $1.7 \mathrm{~m}$ ) and vertical columnar jointing ( 0.5 to $2 \mathrm{~m}$ ) of basalt. The studied xenoliths (size: $\sim 4 \mathrm{~cm}$ ) were sampled within these upper basaltic units at the $S W$ of Hosséré Dammougalré. The Hosséré Dammougalré (altitude: $\sim 1600 \mathrm{~m}$ a.s.l) is a neck of needle-shaped lava which have circular basis (diameter: $\approx 200 \mathrm{~m}$ ) and more or less steep slopes $\left(45^{\circ}-90^{\circ}\right)$. This formation is strongly prismatic and dismantled into blocks, disseminated in chaos on the soil. However, the Hosséré Doro is a dome bounded by small steep cliffs $(20-40 \mathrm{~m}$ high) and the lava blocks are accumulated to the foothill.

\section{Analytical Methods}

The mineral phases were analyzed in polished thin sections with the electron microprobe analyzer using a Cameca microprobe SX100 at the "Université Pierre et Marie Curie", Paris VI (France). The standards data used for analysis are from natural ( $\mathrm{Si}, \mathrm{Al}$ and $\mathrm{K}$ on orthoclase, $\mathrm{Ca}$ on anorthite, $\mathrm{Na}$ on albite, $\mathrm{P}$ on apatite, $\mathrm{Zr}$ on zircon) and synthetic $\left(\mathrm{Fe}\right.$ on $\mathrm{Fe}_{2} \mathrm{O}_{3}, \mathrm{Ba}$ on $\mathrm{BaO}_{4}, \mathrm{Sr}$ on $\mathrm{SrSiO}_{3}$ )

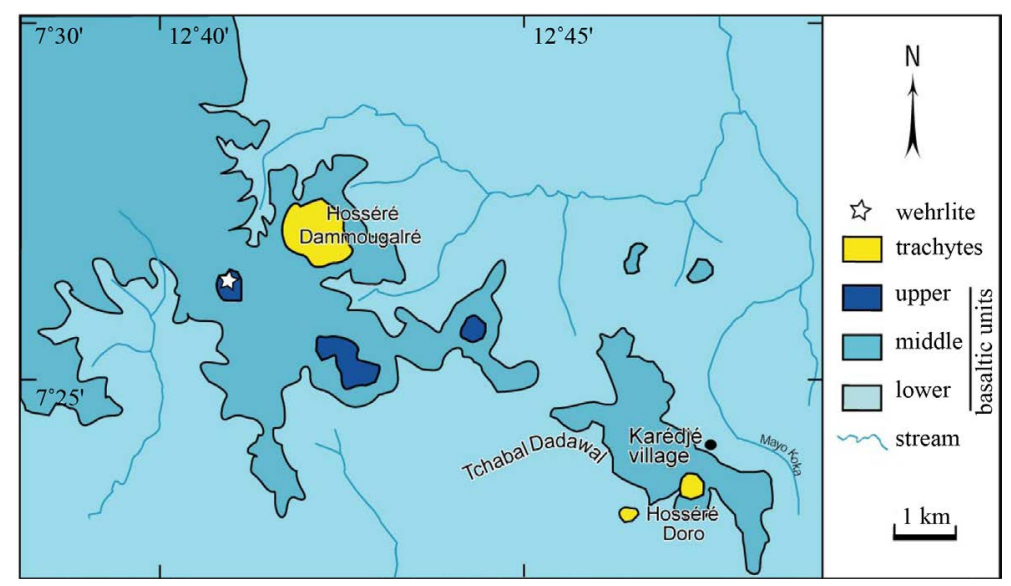

Figure 2. Sketch of the geological map of the studied zone. 
phases.

The measurements were carried out with a beam size of $10-100 \mu \mathrm{m}$, under the following conditions expressed in $\mathrm{kV}$ (accelerating voltage), $\mathrm{nA}$ (beam current) and s (counting times at the peak): Olivine ( $15 \mathrm{kV}, 40 \mathrm{nA}, 20 \mathrm{~s}$ for all elements, except $\mathrm{Si}(10 \mathrm{~s})$ ), clinopyroxene ( $15 \mathrm{kV}, 40 \mathrm{nA}, 20 \mathrm{~s}$ for $\mathrm{Si}, \mathrm{Al}, \mathrm{Fe}, \mathrm{Mg}, \mathrm{Ca}$, $\mathrm{Na}, \mathrm{Mn}$ and $30 \mathrm{~s}$ for $\mathrm{Ti}$ and $\mathrm{Zr}$ ), feldspar ( $15 \mathrm{kV}, 10 \mathrm{nA}, 5 \mathrm{~s}$ for all elements), FeTi oxides and spinel ( $15 \mathrm{kV}, 40 \mathrm{nA}, 40 \mathrm{~s}$ for Ti, Fe, Mn, Mg, $10 \mathrm{~s}$ for Si, $15 \mathrm{~s}$ for $\mathrm{Cr}$ and $30 \mathrm{~s}$ for $\mathrm{Al}$ ). Measurements correction was carried out using the "PAP" program [9].

\section{Results}

\subsection{Petrography}

Host Basalt is characterized by a porphyritic texture. The phenocrysts of olivine $(\sim 2 \mathrm{~mm})$, clinopyroxene $(1.2-2.1 \mathrm{~mm})$ and $\mathrm{Fe}-\mathrm{Ti}$ oxides $(0.5-0.6 \mathrm{~mm})$ are scattered in a groundmass consisting of clinopyroxene, Fe-Ti oxides and plagioclase microlites. Olivine phenocrysts are subhedral, cracked and their edges are sometimes destabilized into iddingsite. Clinopyroxene phenocrysts are euhedral and twinned.

Wehrlite xenoliths ( $4 \mathrm{~cm}$ size) are characterized by an allotriomorphic texture, with inequigranular olivine ( $\sim 40$ vol.\%), clinopyroxene ( $\sim 55 \mathrm{vol} . \%)$ and spinel ( $\sim 5$ vol.\%) crystals. Spinel crystals are red brown and interstitial between the crystals of olivine and clinopyroxene. The contact between the host basalt and wehrlite xenoliths is materialized by the accumulation of small Fe-Ti oxides and plagioclase crystals or the tiny host basalt veins.

\subsection{Mineral Chemistry}

\subsubsection{Olivine}

In the host basalt, forsterite (Fo) values calculated for the analyzed olivine phenocrysts (Table 1) reach $73-74$ and $68-72$, respectively for the core and the rim. The $\mathrm{CaO}$ contents are high (rim, up to $0.43 \mathrm{wt} \%$ and core, up to $0.41 \mathrm{wt} \%$ ). Using bulk-rock composition as a liquid at the total pressure of $1 \mathrm{~atm}$, the crystallization temperatures were estimated after [10] for the core and the rim respectively to $1224^{\circ} \mathrm{C} \pm 60^{\circ} \mathrm{C}$ and $1166^{\circ} \mathrm{C} \pm 48^{\circ} \mathrm{C}$.

For wehrlite xenoliths, the forsterite component (Table 1) range from 76 to 88. These values are similar to those calculated for the wehrlites from the Mount Cameroun $\left(\mathrm{Fo}_{82-86} ;[11]\right)$. $\mathrm{NiO}$ contents reaching $0.19 \mathrm{wt} \%$ and $\mathrm{CaO}$ up to 0.39 wt\%.

\subsubsection{Clinopyroxene}

The clinopyroxene phenocrysts (Table 2) of the host basalt are both diopside and augite (after the nomenclature of [12]; Figure 3). Their $\mathrm{Cr}_{2} \mathrm{O}_{3}$ contents range from $0 \mathrm{wt} \%$ to $0.38 \mathrm{wt} \%$ ), while $\mathrm{TiO}_{2}$ and $\mathrm{Al}_{2} \mathrm{O}_{3}$ contents are high (up to $3.9 \mathrm{wt} \%$ and $8.7 \mathrm{wt} \%$ respectively). The high contents of $\mathrm{Ti}$ and $\mathrm{Al}^{\mathrm{iv}}$ (apfu: atom 
per formula unit) in diopside and augite are linked to a low-pressure of crystallization [13].

Wehrlites contain also diopside and augite, but poor in $\mathrm{Al}_{2} \mathrm{O}_{3}(1.8-5.1 \mathrm{wt} \%)$, $\mathrm{TiO}_{2}(0.8-1.7 \mathrm{wt} \%)$ and rich in $\mathrm{Cr}_{2} \mathrm{O}_{3}$ (up to $\left.0.59 \mathrm{wt} \%\right)$ with $\mathrm{Cr} \#(=100 \times$ atomic $\mathrm{Cr} /(\mathrm{Cr}+\mathrm{Al}))$ up to 15.6 .

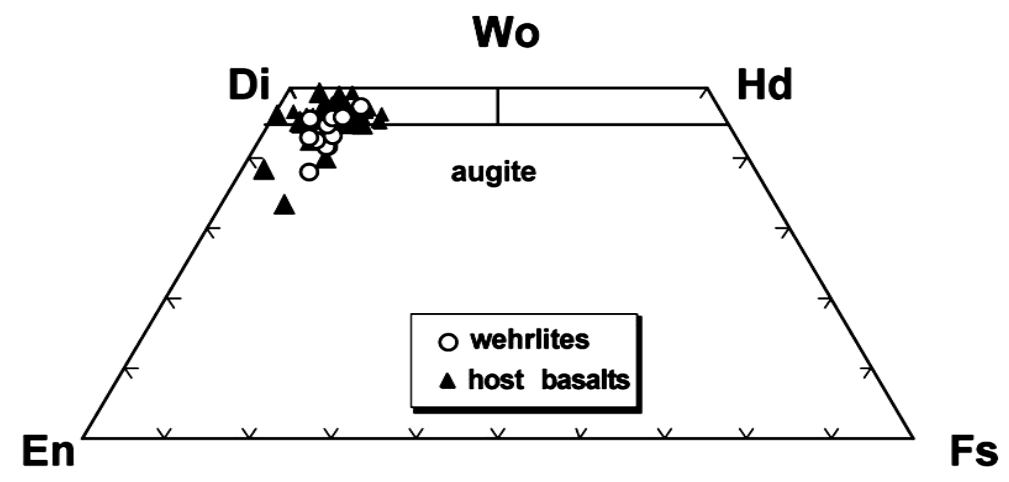

Figure 3. Wo-En-Fs ternary diagram (after Morimoto, 1989) for the classification of clinopyroxene from the studied rocks.

Table 1. Representative compositions of olivine of the host basalt and the studied wehrlite.

\begin{tabular}{|c|c|c|c|c|c|c|c|c|c|c|c|c|c|c|}
\hline \multirow{3}{*}{$\begin{array}{c}\text { rocks } \\
\text { sample nb. } \\
\text { description }\end{array}$} & \multicolumn{2}{|c|}{ host basalt } & \multirow[b]{2}{*}{132} & \multirow[b]{2}{*}{132} & \multirow[b]{2}{*}{132} & \multicolumn{3}{|c|}{ wehrlite } & \multirow[b]{2}{*}{$132-1$} & \multirow[b]{2}{*}{$132-1$} & \multirow[b]{2}{*}{$132-1$} & \multirow[b]{2}{*}{$132-1$} & \multirow[b]{2}{*}{$132-1$} & \multirow[b]{2}{*}{$132-1$} \\
\hline & 132 & 132 & & & & 132 & $132-1$ & $132-1$ & & & & & & \\
\hline & $\mathrm{ph}, \mathrm{r}$ & $\mathrm{ph}, \mathrm{c}$ & $\mathrm{ph}, \mathrm{c}$ & $\mathrm{ph}, \mathrm{c}$ & $\mathrm{ph}, \mathrm{c}$ & $\mathrm{ph}, \mathrm{r}$ & & & & & & & & \\
\hline $\mathrm{SiO}_{2}(\mathrm{wt} \%)$ & 37.02 & 37.09 & 37.07 & 37.15 & 36.62 & 37.98 & 38.27 & 37.43 & 38.39 & 38.99 & 38.86 & 37.31 & 36.36 & 36.74 \\
\hline $\mathrm{Al}_{2} \mathrm{O}_{3}$ & 0.10 & 0.06 & 0.00 & 0.00 & 0.11 & 0.04 & 0.01 & 0.02 & 0.00 & 0.07 & 0.09 & 0.05 & 0.00 & 0.00 \\
\hline $\mathrm{FeO}$ & 27.25 & 24.15 & 24.50 & 23.99 & 24.69 & 25.29 & 18.58 & 19.22 & 19.46 & 15.09 & 15.71 & 15.95 & 22.86 & 23.62 \\
\hline $\mathrm{MnO}$ & 0.92 & 0.58 & 0.60 & 0.60 & 0.40 & 0.57 & 0.37 & 0.20 & 0.42 & 0.05 & 0.17 & 0.21 & 0.53 & 0.51 \\
\hline $\mathrm{MgO}$ & 32.93 & 36.18 & 36.33 & 36.51 & 36.52 & 35.87 & 41.84 & 41.33 & 41.78 & 44.54 & 44.73 & 44.81 & 37.83 & 37.30 \\
\hline $\mathrm{CaO}$ & 0.30 & 0.41 & 0.39 & 0.38 & 0.36 & 0.43 & 0.30 & 0.29 & 0.32 & 0.15 & 0.17 & 0.15 & 0.36 & 0.39 \\
\hline $\mathrm{NiO}$ & 0.09 & 0.16 & 0.12 & 0.09 & 0.04 & 0.11 & 0.04 & 0.05 & 0.15 & 0.17 & 0.11 & 0.19 & 0.00 & 0.08 \\
\hline Total & 98.73 & 98.64 & 99.00 & 98.73 & 98.74 & 100.28 & 99.41 & 98.55 & 100.50 & 99.05 & 99.83 & 98.67 & 97.93 & 98.64 \\
\hline Si (apfu) & 1.008 & 0.990 & 0.987 & 0.990 & 0.976 & 1.002 & 0.982 & 0.971 & 0.978 & 0.988 & 0.978 & 0.949 & 0.968 & 0.975 \\
\hline $\mathrm{Al}$ & 0.003 & 0.002 & 0.000 & 0.000 & 0.003 & 0.001 & 0.000 & 0.001 & 0.000 & 0.002 & 0.003 & 0.001 & 0.000 & 0.000 \\
\hline $\mathrm{Fe}^{2+}$ & 0.620 & 0.522 & 0.518 & 0.513 & 0.505 & 0.558 & 0.364 & 0.359 & 0.371 & 0.297 & 0.289 & 0.237 & 0.444 & 0.475 \\
\hline $\mathrm{Mn}$ & 0.021 & 0.013 & 0.013 & 0.014 & 0.009 & 0.013 & 0.008 & 0.004 & 0.009 & 0.001 & 0.004 & 0.004 & 0.012 & 0.011 \\
\hline $\mathrm{Mg}$ & 1.337 & 1.440 & 1.441 & 1.450 & 1.451 & 1.411 & 1.601 & 1.598 & 1.587 & 1.682 & 1.678 & 1.699 & 1.501 & 1.476 \\
\hline $\mathrm{Ca}$ & 0.009 & 0.012 & 0.011 & 0.011 & 0.010 & 0.012 & 0.008 & 0.008 & 0.009 & 0.004 & 0.005 & 0.004 & 0.010 & 0.011 \\
\hline $\mathrm{Ni}$ & 0.002 & 0.003 & 0.002 & 0.002 & 0.001 & 0.002 & 0.001 & 0.001 & 0.003 & 0.003 & 0.002 & 0.004 & 0.000 & 0.002 \\
\hline Fo (\%) & 68 & 73 & 74 & 74 & 74 & 72 & 81 & 82 & 81 & 85 & 85 & 88 & 77 & 76 \\
\hline $\mathrm{Fa}$ & 32 & 27 & 26 & 26 & 26 & 28 & 19 & 18 & 19 & 15 & 15 & 12 & 23 & 24 \\
\hline $\mathrm{mg \#}$ & 68.30 & 73.41 & 73.56 & 73.84 & 74.17 & 71.66 & 81.49 & 81.65 & 81.07 & 84.99 & 85.29 & 87.74 & 77.16 & 75.65 \\
\hline
\end{tabular}

ph.r: phenocryst. rim; ph.c: phenocryst core. 
Table 2. Representative compositions of clinopyroxene of the host basalt and the studied wehrlite.

\begin{tabular}{|c|c|c|c|c|c|c|c|c|c|c|c|c|c|c|c|c|c|c|c|}
\hline \multirow{2}{*}{$\begin{array}{c}\text { rocks } \\
\text { sample nb. }\end{array}$} & \multicolumn{2}{|c|}{ host basalt } & \multicolumn{6}{|c|}{ wehrlite } & \multirow[b]{2}{*}{$132-1$} & \multirow[b]{2}{*}{$132-1$} & \multirow[b]{2}{*}{$132-1$} & \multirow[b]{2}{*}{$132-1$} & \multirow[b]{2}{*}{$132-1$} & \multirow[b]{2}{*}{$132-1$} & \multirow[b]{2}{*}{$132-1$} & \multirow[b]{2}{*}{$132-1$} & \multirow[b]{2}{*}{$132-1$} & \multirow[b]{2}{*}{$132-1$} & \multirow[b]{2}{*}{$132-1$} \\
\hline & 132 & 132 & 132 & 132 & $132-1$ & $132-1$ & $132-1$ & $132-1$ & & & & & & & & & & & \\
\hline $\mathrm{SiO}_{2}(\mathrm{wt} \%)$ & 44.07 & 47.59 & 50.66 & 47.01 & 51.06 & 51.80 & 51.60 & 50.69 & 50.90 & 51.93 & 50.99 & 53.18 & 51.14 & 50.43 & 52.17 & 50.29 & 47.72 & 50.86 & 51.10 \\
\hline $\mathrm{TiO}_{2}$ & 3.91 & 1.98 & 0.65 & 1.95 & 0.89 & 1.78 & 0.97 & 1.03 & 0.99 & 0.93 & 0.94 & 1.19 & 1.21 & 0.92 & 0.83 & 1.16 & 3.04 & 1.61 & 0.93 \\
\hline $\mathrm{Al}_{2} \mathrm{O}_{3}$ & 8.75 & 7.23 & 5.27 & 8.29 & 4.60 & 4.33 & 3.20 & 4.97 & 4.65 & 4.87 & 4.76 & 1.85 & 3.86 & 5.19 & 4.76 & 3.73 & 5.30 & 3.20 & 4.70 \\
\hline $\mathrm{Cr}_{2} \mathrm{O}_{3}$ & 0.00 & 0.06 & 0.25 & 0.38 & 0.34 & 0.55 & 0.46 & 0.43 & 0.37 & 0.50 & 0.30 & 0.51 & 0.53 & 0.59 & 0.43 & 0.51 & 0.00 & 0.49 & 0.47 \\
\hline $\mathrm{FeO}$ & 6.92 & 6.68 & 6.90 & 6.21 & 5.46 & 4.91 & 4.53 & 5.73 & 5.13 & 4.91 & 5.23 & 4.46 & 5.24 & 5.07 & 5.04 & 5.85 & 7.30 & 5.27 & 5.46 \\
\hline $\mathrm{MnO}$ & 0.32 & 0.21 & 0.15 & 0.17 & 0.13 & 0.13 & 0.11 & 0.12 & 0.11 & 0.04 & 0.02 & 0.11 & 0.23 & 0.16 & 0.04 & 0.05 & 0.00 & 0.25 & 0.08 \\
\hline $\mathrm{MgO}$ & 11.56 & 13.18 & 17.67 & 13.84 & 15.52 & 15.00 & 15.89 & 16.36 & 15.31 & 15.12 & 15.54 & 16.27 & 15.50 & 15.29 & 15.35 & 14.64 & 12.68 & 14.86 & 15.00 \\
\hline $\mathrm{CaO}$ & 22.34 & 21.49 & 17.18 & 21.21 & 21.17 & 20.77 & 22.17 & 18.42 & 20.55 & 20.66 & 20.52 & 21.53 & 20.93 & 20.65 & 20.01 & 21.97 & 22.38 & 22.01 & 21.05 \\
\hline $\mathrm{Na}_{2} \mathrm{O}$ & 0.49 & 0.60 & 0.68 & 0.56 & 0.79 & 0.59 & 0.62 & 0.82 & 0.89 & 0.85 & 0.81 & 0.71 & 0.70 & 0.84 & 0.72 & 0.57 & 0.62 & 0.47 & 0.77 \\
\hline Total & 98.37 & 99.02 & 99.40 & 99.60 & 99.94 & 99.85 & 99.55 & 98.57 & 98.91 & 99.80 & 99.12 & 99.81 & 99.33 & 99.12 & 99.35 & 98.75 & 99.04 & 99.01 & 99.57 \\
\hline $\begin{array}{l}\mathrm{Fe}_{2} \mathrm{O}_{3} \\
\text { (calc.) }\end{array}$ & 2.91 & 0.79 & 2.84 & 3.14 & 0.97 & 0.00 & 1.84 & 0.97 & 1.40 & 0.00 & 1.28 & 0.00 & 1.01 & 1.88 & 0.00 & 1.77 & 1.81 & 0.13 & 0.00 \\
\hline $\mathrm{FeO}$ (calc.) & 4.31 & 5.97 & 4.35 & 3.38 & 4.59 & 4.91 & 2.88 & 4.86 & 3.88 & 4.91 & 4.07 & 4.46 & 4.33 & 3.38 & 5.04 & 4.26 & 5.67 & 5.15 & 5.46 \\
\hline Total (calc.) & 98.66 & 99.10 & 99.69 & 99.91 & 100.04 & 99.88 & 99.76 & 98.67 & 99.05 & 99.87 & 99.26 & 99.81 & 99.45 & 99.35 & 99.40 & 98.93 & 99.22 & 99.06 & 99.57 \\
\hline Si (apfu) & 1.670 & 1.793 & 1.850 & 1.736 & 1.885 & 1.907 & 1.894 & 1.873 & 1.879 & 1.901 & 1.877 & 1.948 & 1.886 & 1.857 & 1.919 & 1.874 & 1.793 & 1.892 & 1.900 \\
\hline $\mathrm{Al}^{\mathrm{IV}}$ & 0.330 & 0.207 & 0.150 & 0.264 & 0.115 & 0.093 & 0.106 & 0.127 & 0.121 & 0.099 & 0.123 & 0.052 & 0.114 & 0.143 & 0.081 & 0.126 & 0.207 & 0.108 & 0.100 \\
\hline $\mathrm{Cr}$ & 0.000 & 0.002 & 0.007 & 0.011 & 0.010 & 0.016 & 0.013 & 0.013 & 0.011 & 0.014 & 0.009 & 0.015 & 0.015 & 0.017 & 0.012 & 0.015 & 0.000 & 0.014 & 0.011 \\
\hline $\mathrm{Fe}^{3+(\mathrm{VI})}$ & 0.083 & 0.022 & 0.078 & 0.087 & 0.027 & 0.000 & 0.051 & 0.027 & 0.039 & 0.000 & 0.035 & 0.000 & 0.028 & 0.052 & 0.000 & 0.050 & 0.051 & 0.004 & 0.000 \\
\hline $\mathrm{Fe}^{2+}$ & 0.136 & 0.188 & 0.133 & 0.104 & 0.142 & 0.151 & 0.088 & 0.150 & 0.120 & 0.150 & 0.125 & 0.136 & 0.134 & 0.104 & 0.155 & 0.133 & 0.178 & 0.160 & 0.170 \\
\hline $\mathrm{Mn}$ & 0.010 & 0.007 & 0.005 & 0.005 & 0.004 & 0.004 & 0.004 & 0.004 & 0.004 & 0.001 & 0.001 & 0.004 & 0.007 & 0.005 & 0.001 & 0.002 & 0.000 & 0.008 & 0.003 \\
\hline $\mathrm{Mg}$ & 0.653 & 0.740 & 0.962 & 0.762 & 0.854 & 0.823 & 0.870 & 0.901 & 0.842 & 0.825 & 0.853 & 0.888 & 0.852 & 0.839 & 0.842 & 0.813 & 0.711 & 0.824 & 0.831 \\
\hline $\mathrm{Ca}$ & 0.907 & 0.827 & 0.672 & 0.839 & 0.798 & 0.819 & 0.871 & 0.729 & 0.813 & 0.810 & 0.809 & 0.845 & 0.827 & 0.815 & 0.789 & 0.877 & 0.901 & 0.877 & 0.799 \\
\hline $\mathrm{Na}$ & 0.036 & 0.044 & 0.048 & 0.040 & 0.056 & 0.042 & 0.044 & 0.059 & 0.064 & 0.060 & 0.058 & 0.050 & 0.050 & 0.060 & 0.051 & 0.041 & 0.045 & 0.034 & 0.055 \\
\hline Wo (\%) & 46.56 & 41.93 & 33.15 & 42.43 & 41.56 & 43.24 & 45.41 & 37.60 & 42.82 & 42.95 & 42.26 & 44.65 & 43.11 & 42.63 & 41.59 & 45.54 & 46.80 & 45.28 & 41.86 \\
\hline En & 44.87 & 46.30 & 59.15 & 51.33 & 50.13 & 47.95 & 49.65 & 53.49 & 50.07 & 48.27 & 50.34 & 47.98 & 49.18 & 51.05 & 49.33 & 46.93 & 42.61 & 45.80 & 48.29 \\
\hline Fs & 8.57 & 11.77 & 7.70 & 6.23 & 8.31 & 8.80 & 4.94 & 8.91 & 7.11 & 8.78 & 7.40 & 7.37 & 7.71 & 6.32 & 9.08 & 7.53 & 10.59 & 8.91 & 9.85 \\
\hline $\mathrm{Ti} / \mathrm{Al}$ & 0.28 & 0.17 & 0.08 & 0.15 & 0.12 & 0.26 & 0.19 & 0.13 & 0.14 & 0.12 & 0.13 & 0.41 & 0.20 & 0.11 & 0.11 & 0.20 & 0.37 & 0.32 & 0.13 \\
\hline $\begin{array}{l}\mathrm{Cr}^{\star} 100 / \\
(\mathrm{Cr}+\mathrm{Al})\end{array}$ & 0.00 & 0.56 & 3.06 & 2.99 & 4.70 & 7.87 & 8.71 & 5.53 & 5.12 & 6.42 & 4.09 & 15.55 & 8.37 & 7.07 & 5.68 & 8.36 & 0.00 & 9.30 & 5.06 \\
\hline
\end{tabular}

According to the $\mathrm{Al}^{\mathrm{vi}}$ vs $\mathrm{Al}^{\mathrm{iv}}$ diagram (Figure 4), these clinopyroxene crystals crystalized at high pressure near of the RMC (Refractory Mantle Clinopyroxene, [14]) field. 


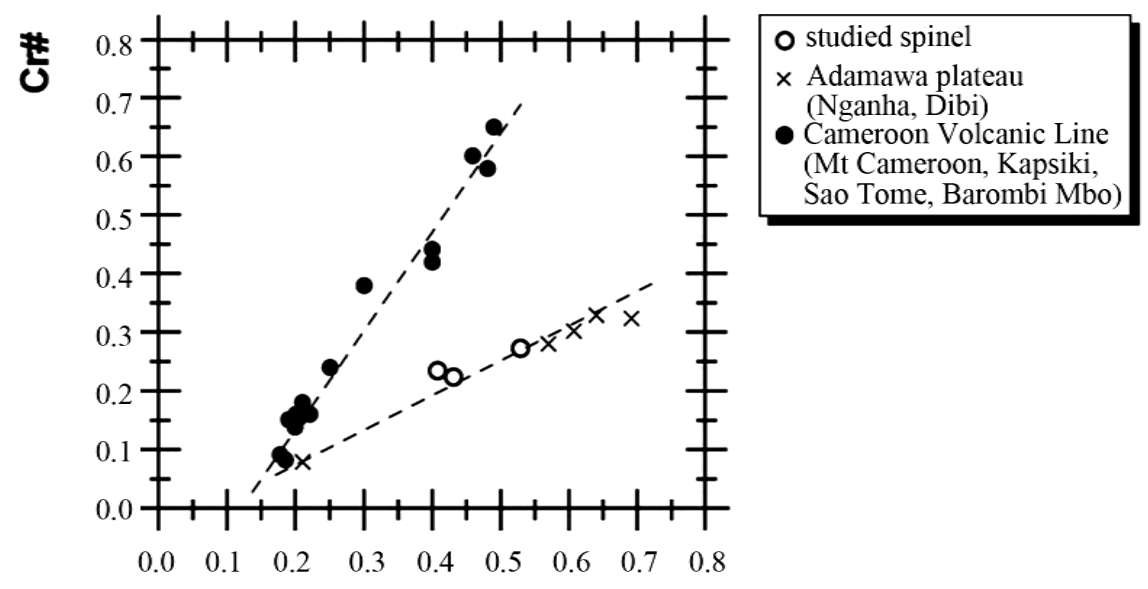

$\mathbf{F e}^{2+}\left(\mathbf{F e}{ }^{2+}+\mathbf{M g}\right)$

Figure 4. Plot of $\mathrm{Cr} \#$ vs. $\mathrm{Fe}^{2+} /\left(\mathrm{Fe}^{2+}+\mathrm{Mg}\right)$ values of the spinel for xenoliths from the SW of the Hosséré Dammougalré and those of the others sector of the Adamawa Plateau and the Cameroon Volcanic Line.

\subsubsection{Fe-Ti Oxides}

The titano-magnetite is present in the host basalt (Table 3), with $\mathrm{TiO}_{2}$ and $\mathrm{FeO}$ contents reaching respectively $25.0 \mathrm{wt} \%$ and $69.5 \mathrm{wt} \%$.

\subsubsection{Spinel}

Spinel of wherlites $\left(\mathrm{Al}>\mathrm{Cr}, \mathrm{Mg}>\mathrm{Fe}^{2+}\right)$ is chromiferous with the values of $\mathrm{Cr} \#$ $\left(\mathrm{Cr} \times 100 /(\mathrm{Cr}+\mathrm{Al})\right.$ and $\mathrm{Fe}^{2+} /\left(\mathrm{Fe}^{2+}+\mathrm{Mg}\right)$ positively correlated (Table 4; Figure 4). The correlation trend displays on the Figure 4, present two distinct evolutions, one for the Adamawa Plateau (Nganha; [15], Dibi; [3]) and another for the Cameroon Volcanic Line (São Tomé; [16], Mbarombi Mbo; [17]), Mount Cameroon; [11] [18] and Kapsiki Plateau; [19]). The distribution of the spinel compositions in Cr-Al-Fe ${ }^{3+}$ ternary diagram (Figure 5) both for Adamawa Plateau and the Cameroon Volcanic Line reflects the heterogeneous nature of the magmatic source. The $\mathrm{Ti}\left(0.44-0.52\right.$ a.p.f.u) and $\mathrm{Fe}^{2+}(3.15$ - 3.59 a.p.f.u) contents are high and could be link to the re-equilibration during the crystallization of the clinopyroxene from the host basalt.

\subsubsection{Plagioclase}

The compositions of plagioclase (Table 5) from the host basalt range from bytownite $\left(\mathrm{An}_{71} \mathrm{Ab}_{28}\right)$ to labradorite $\left(\mathrm{An}_{61-69} \mathrm{Ab} \mathrm{b}_{37-30}\right)$.

\section{Discussion}

\subsection{Origin of Wehrlites}

Ultramafic xenoliths are considered as fragments of lithospheric mantle [1] or the residues of the partial melting of mantle [20].

Clinopyroxenes in the studied wehrlites are low in $\mathrm{TiO}_{2}(0.8-1.7 \mathrm{wt} \%)$ and $\mathrm{Al}_{2} \mathrm{O}_{3}$ (1.8 - $\left.5.1 \mathrm{wt} \% 3-4 \mathrm{wt} \%\right)$ as typical for clinopyroxenes of residual mantle origin [14] [13]. The refractory elements as $\mathrm{Cr}$ are enriched in the residue, as 


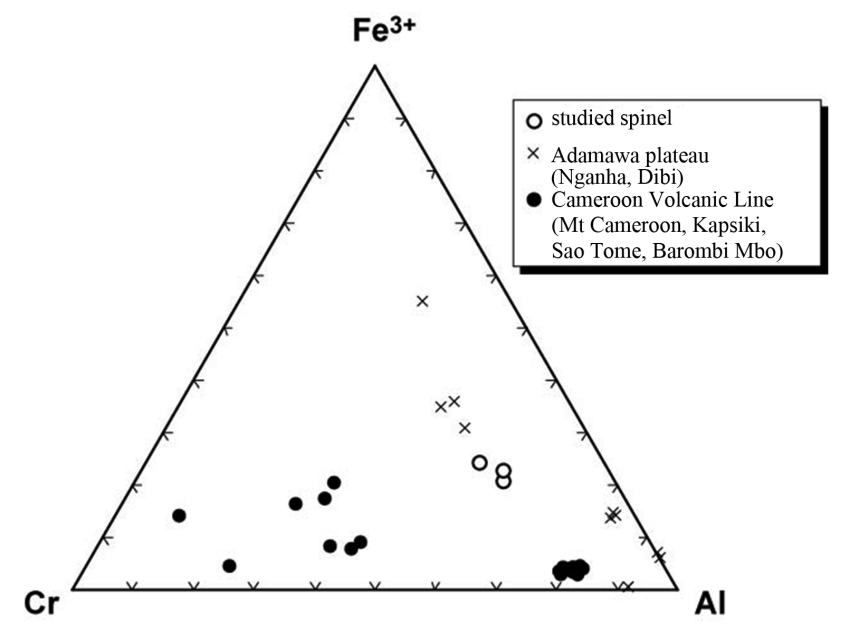

Figure 5. $\mathrm{Fe}^{3+}-\mathrm{Cr}-\mathrm{Al}$ ternary diagram of the spinel for xenoliths from the SW of the Hosséré Dammougalré and those of the others sector of the Adamawa Plateau and the Cameroon Volcanic Line.

Table 3. Representative compositions of Fe-Ti oxides of host basalt.

\begin{tabular}{|c|c|c|c|c|c|c|c|}
\hline sample nb. & 132 & 132 & 132 & 132 & 132 & 132 & 132 \\
\hline $\mathrm{SiO}_{2}(\mathrm{wt} \%)$ & 0.00 & 0.00 & 0.05 & 0.03 & 0.13 & 1.01 & 0.07 \\
\hline $\mathrm{TiO}_{2}$ & 24.63 & 24.66 & 25.03 & 24.56 & 19.60 & 19.59 & 17.83 \\
\hline $\mathrm{Al}_{2} \mathrm{O}_{3}$ & 2.64 & 2.85 & 2.35 & 2.02 & 2.41 & 1.51 & 5.68 \\
\hline $\mathrm{Cr}_{2} \mathrm{O}_{3}$ & 0.07 & 0.21 & 0.11 & 0.17 & 0.50 & 0.05 & 0.00 \\
\hline $\mathrm{FeO}$ & 65.52 & 63.68 & 65.83 & 65.83 & 67.46 & 69.58 & 67.68 \\
\hline $\mathrm{MnO}$ & 0.74 & 0.83 & 0.74 & 0.86 & 0.76 & 2.33 & 0.64 \\
\hline $\mathrm{MgO}$ & 3.93 & 4.51 & 3.57 & 3.28 & 4.02 & 1.43 & 4.13 \\
\hline $\mathrm{CaO}$ & 0.24 & 0.09 & 0.06 & 0.13 & 0.11 & 0.22 & 0.00 \\
\hline Total & 97.77 & 96.84 & 97.72 & 96.87 & 95.18 & 96.08 & 96.19 \\
\hline \multicolumn{8}{|c|}{ Ilmenite basis } \\
\hline $\mathrm{Fe}_{2} \mathrm{O}_{3}$ (calc.) & 36.69 & 35.85 & 36.11 & 36.37 & 41.49 & 40.88 & 41.21 \\
\hline $\mathrm{FeO}$ (calc.) & 32.51 & 31.42 & 33.34 & 33.11 & 30.13 & 32.80 & 29.60 \\
\hline Total (calc.) & 101.44 & 100.43 & 101.34 & 100.51 & 99.14 & 99.82 & 99.16 \\
\hline \multicolumn{8}{|c|}{ Ulvospinel basis } \\
\hline $\mathrm{Fe}_{2} \mathrm{O}_{3}$ (calc.) & 20.27 & 19.41 & 19.39 & 19.98 & 28.31 & 26.93 & 29.93 \\
\hline $\mathrm{FeO}$ (calc.) & 47.28 & 46.21 & 48.38 & 47.86 & 41.98 & 45.35 & 39.75 \\
\hline Total (calc.) & 99.80 & 98.78 & 99.67 & 98.87 & 97.82 & 98.42 & 98.03 \\
\hline Si (apfu) & 0.000 & 0.001 & 0.014 & 0.008 & 0.037 & 0.303 & 0.021 \\
\hline $\mathrm{Ti}$ & 5.343 & 5.374 & 5.457 & 5.418 & 4.346 & 4.400 & 3.704 \\
\hline $\mathrm{Al}$ & 0.898 & 0.973 & 0.803 & 0.698 & 0.836 & 0.531 & 1.960 \\
\hline $\mathrm{Cr}$ & 0.017 & 0.049 & 0.025 & 0.039 & 0.117 & 0.011 & 0.000 \\
\hline $\mathrm{Fe}^{3+}$ & 4.399 & 4.231 & 4.231 & 4.411 & 6.281 & 6.051 & 6.591 \\
\hline $\mathrm{Fe}^{2+}$ & 11.402 & 11.194 & 11.729 & 11.739 & 10.350 & 11.327 & 9.728 \\
\hline $\mathrm{Mn}$ & 0.180 & 0.204 & 0.180 & 0.212 & 0.190 & 0.590 & 0.159 \\
\hline $\mathrm{Mg}$ & 1.689 & 1.947 & 1.542 & 1.434 & 1.766 & 0.638 & 1.800 \\
\hline $\mathrm{Ca}$ & 0.073 & 0.028 & 0.020 & 0.041 & 0.034 & 0.069 & 0.000 \\
\hline Usp (\%) & 68.09 & 68.64 & 69.59 & 68.89 & 55.90 & 59.46 & 48.54 \\
\hline
\end{tabular}


Table 4. Representative compositions of the spinel of the studied wehrlite.

\begin{tabular}{|c|c|c|c|}
\hline sample nb. & $132-1$ & $132-1$ & $132-1$ \\
\hline $\mathrm{TiO}_{2}(w t \%)$ & 2.92 & 2.48 & 2.85 \\
\hline $\mathrm{Al}_{2} \mathrm{O}_{3}$ & 32.62 & 32.64 & 28.78 \\
\hline $\mathrm{Cr}_{2} \mathrm{O}_{3}$ & 14.90 & 14.26 & 16.15 \\
\hline $\mathrm{FeO}$ & 33.62 & 35.77 & 39.57 \\
\hline $\mathrm{MnO}$ & 0.32 & 0.23 & 0.50 \\
\hline $\mathrm{MgO}$ & 14.11 & 13.65 & 10.84 \\
\hline $\mathrm{CaO}$ & 0.01 & 0.00 & 0.06 \\
\hline Total & 98.73 & 99.04 & 98.74 \\
\hline $\mathrm{Fe}_{2} \mathrm{O}_{3}(\mathrm{calc})$ & 17.82 & 19.36 & 19.58 \\
\hline $\mathrm{FeO}$ (calc) & 17.58 & 18.34 & 21.95 \\
\hline Total (calc) & 100.52 & 100.98 & 100.7 \\
\hline $\mathrm{Ti}(\mathrm{apfu})$ & 0.516 & 0.439 & 0.522 \\
\hline $\mathrm{Al}$ & 9.042 & 9.044 & 8.259 \\
\hline $\mathrm{Cr}$ & 2.771 & 2.651 & 3.109 \\
\hline $\mathrm{Fe}^{3+}$ & 3.154 & 3.426 & 3.588 \\
\hline $\mathrm{Fe}^{2+}$ & 3.458 & 3.607 & 4.471 \\
\hline $\mathrm{Mn}$ & 0.063 & 0.046 & 0.102 \\
\hline $\mathrm{Mg}$ & 4.949 & 4.786 & 3.934 \\
\hline $\mathrm{Ca}$ & 0.003 & 0.000 & 0.015 \\
\hline
\end{tabular}

Table 5. Representative compositions of the plagioclase of the host basalt.

\begin{tabular}{|c|c|c|c|c|c|c|}
\hline sample nb. & 132 & 132 & 132 & 132 & 132 & 132 \\
\hline $\mathrm{SiO}_{2}(\mathrm{wt} \%)$ & 50.71 & 50.67 & 52.08 & 49.4 & 50.63 & 51.66 \\
\hline $\mathrm{Al}_{2} \mathrm{O}_{3}$ & 28.40 & 30.51 & 30.31 & 32.34 & 30.66 & 31.25 \\
\hline $\mathrm{FeO}$ & 1.30 & 0.70 & 0.55 & 0.39 & 0.54 & 0.68 \\
\hline $\mathrm{CaO}$ & 12.93 & 12.4 & 12.07 & 14.11 & 13.55 & 13.86 \\
\hline $\mathrm{Na}_{2} \mathrm{O}$ & 4.12 & 3.84 & 4.13 & 3.20 & 3.25 & 3.30 \\
\hline $\mathrm{K}_{2} \mathrm{O}$ & 0.66 & 0.34 & 0.34 & 0.15 & 0.25 & 0.24 \\
\hline Total & 98.12 & 98.46 & 99.48 & 99.57 & 98.88 & 100.98 \\
\hline Si (apfu) & 2.319 & 2.335 & 2.371 & 2.26 & 2.331 & 2.331 \\
\hline $\mathrm{Al}$ & 1.531 & 1.657 & 1.626 & 1.744 & 1.664 & 1.661 \\
\hline $\mathrm{Fe}^{2+}$ & 0.000 & 0.027 & 0.021 & 0.015 & 0.021 & 0.026 \\
\hline $\mathrm{Ca}$ & 0.634 & 0.612 & 0.589 & 0.691 & 0.668 & 0.670 \\
\hline $\mathrm{Na}$ & 0.366 & 0.344 & 0.366 & 0.284 & 0.291 & 0.289 \\
\hline $\mathrm{K}$ & 0.038 & 0.020 & 0.02 & 0.009 & 0.015 & 0.014 \\
\hline An (\%) & 64 & 64 & 61 & 71 & 69 & 69 \\
\hline $\mathrm{Ab}$ & 32 & 34 & 37 & 28 & 30 & 30 \\
\hline Or & 3 & 2 & 2 & 1 & 2 & 1 \\
\hline
\end{tabular}


demonstrated for the Cr-rich clinopyroxene from the studied xenoliths. Thus, the studied spinel-bearing wehrlite xenoliths, characterized by the presence of Cr-diopside, represent probably the residual portions of the upper mantle [21]. The range of Ni contents (up to $1900 \mathrm{ppm}$ ) of olivine of wehrlites from the SW of Hosséré Dammougalré confirms that they are residues of melting. However, the compositions of olivine crystals which are characterized by the forsterite contents lower than 90\%, may, have been modified by Fe-Mg equilibration between wehrlite olivine and the host basalt. Re-equilibration between Fe-Mg and host basalt at low temperatures is corroborated by slight similarity between wehrlite olivine $\mathrm{Mg} \#$ (Fo76 - 88), $\mathrm{NiO}$ (0.04 - 0.19 wt.) and $\mathrm{CaO}(0.15$ - 0.39) contents and olivine in host basalt $\mathrm{Mg} \#$ (Fo68 - 74), $\mathrm{NiO}(0.04-0.16 \mathrm{wt} \%)$ and $\mathrm{CaO}$ (0.30 - 0.43). Thus, the occurrence of small plagioclase and oxides crystal surrounding the wehrlites indicates that olivine re-equilibration took place at low pressures $(<0.2 \mathrm{GPa}$; $[22])$.

\subsection{Geothermometry and Geobarometry}

Equilibrium temperatures calculated from $\mathrm{Fe} / \mathrm{Mg}$ exchange reaction for olivine/ spinel using geothermometers [23] vary between $944^{\circ} \mathrm{C}$ and $1102^{\circ} \mathrm{C}$. These temperatures are near of that estimated at liquidus above $1150^{\circ} \mathrm{C}$ after [24] for the crystallization of the Cr-spinel.

The $\mathrm{CaO}$ contents $(0.15-0.39 \mathrm{wt} \%)$ of the studied wehrlite olivine are obviously higher than that of olivine of mantle origin $(0.05 \%-0.1 \%$ : [17]), which involve low pressure $(<0.2 \mathrm{GPa}$; [22]) equilibrium environment, probably near the surface. However, as evidenced by the $\mathrm{Al}^{\mathrm{vi}} v s \mathrm{Al}^{\mathrm{liv}}$ diagram (see Figure 6) all the analyzed wehrlite clinopyroxenes have crystalized at high pressures [13] near of the RMC (Refractory Mantle Clinopyroxene; [14]) field. These contrasted results, would indicate that the wehrlite clinopyroxene would have been re-equilibrated in the host basalt at high pressures (shallow depths) during their as

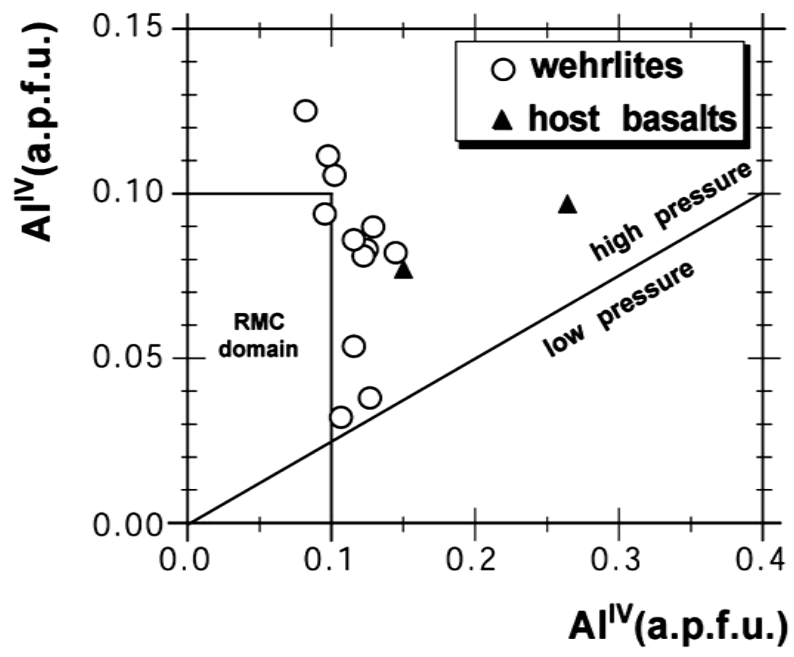

Figure 6. $\mathrm{Al}^{\mathrm{VI}}$ vs. $\mathrm{Al}^{\mathrm{IV}}$ (apfu) plot of clinopyroxene from ultramafic xenoliths and host basalt. Field for refractory mantle clinopyroxenes (RMC) after Jagoutz et al. (1979). 
cending towards the surface. As estimated for Ngao Voglar xenoliths [5], which are situated in the same geological domain (Adamawa Plateau), the wehrlite xenoliths from the SW of Hosséré Dammougalré have likely been entrained by host basalt and carried up to the earth's surface from the depth limit of 57 - 58 $\mathrm{km}$.

\section{Conclusion}

Petrographical, mineral chemistry and thermobarometric data indicated that the wehrlite xenoliths from the SW of Hosséré Dammougalré are likely residual portions of the mantle partial melting, strongly re-equilibrated by the host melt which were extracted from shallow depths $(\sim 60 \mathrm{~km})$ at temperatures estimated around $1100^{\circ} \mathrm{C}$.

\section{Acknowledgements}

The French "Ministère de la Coopération" is acknowledged for providing a grants to I.B.G.M. for nine-month stay in France at the "Laboratoire de magmatologie et de géochimie inorganique et expérimentale, Université Pierre-et-Marie-Curie”, Paris. Pr Bernard Déruelle is acknowledged for his assistance and his supervision during the laboratory-work at this university.

\section{References}

[1] Menzies, M., Rodgers, N., Tindle, A. and Hawkesworth, C. (1987) Metasomatic and Enrichment Processes in Lithospheric Peridotites, an Effect of the Asthenosphere-Lithosphere Interaction. In: Menzies, M. and Hawkesworth, C.J., Eds., Mantle Metasomatism Academic Press, London, 313-361.

[2] Girod, M., Dautria, J.-M., Ball, E. and Soba, D. (1984) Estimation of the Depth of Moho under the Volcanic Massif of Adamaoua (Cameroon), from the Study of Enclaves of Lherzolite. Comptes Rendus de l'Académie des Sciences Paris, Série II, 298, 699-704.

[3] Dautria, J.-M. and Girod, M. (1986) The Enclaves of the Spinel and Plagioclase Lherzolite of the Dibi Volcano (Adamaoua, Cameroon): Witnesses of an Abnormal upper Mantle. Bulletin de Minéralogie, 109, 275-286.

[4] Temdjim, R. (2005) Contribution to the Knowledge of the upper Mantle of Cameroon through the Study of the Ultrabasic and Basic Enclaves by the Volcanoes of Youkou (Adamaoua) and Nyos (Cameruon Line). Thèse de Doctorat d'Etat, Université de Yaoundé I, Yaounde, 339.

[5] Nkouandou, O.F. and Temdjim, R. (2011) Petrology of Spinel Lherzolite Xenoliths and Host Basaltic Lava from Ngao Voglar Volcano, Adamawa Massif (Cameroon Volcanic Line, West Africa): Equilibrium Conditions and Mantle Characteristics. Journal of Geoscience, 56, 375-387.

[6] Nguihdama, D., Chazot, G., Kamgang, P., Mbowou. G.I.B. and Ngounouno, I. (2014) Spinel-Bearing Lherzolite Xenoliths from Hosséré Garba (Likok, Adamawa-Cameroon): Mineral Compositions and Geothermobarometric Implications. International Journal of Geosciences, 5, 1435-1444.

https://doi.org/10.4236/ijg.2014.512117

[7] Moreau, C., Regnoult, J.-M., Déruelle, B. and Robineau, B. (1987) A New Tectonic 
Model for the Cameroon Line, Central Africa. Tectonophysics, 139, 317-334. https://doi.org/10.1016/0040-1951(87)90206-X

[8] Cornacchia, M. and Dars, R. (1983) Un trait structural majeur du continent africain: les linéaments centrafricains du Cameroun au golfe d'Aden [A Major Structural Feature of the African Continent: The Cameroonian Lineaments to the Gulf of Aden]. Bulletin de la Société Géologique de France, 25, 101-109.

https://doi.org/10.2113/gssgfbull.S7-XXV.1.101

[9] Pouchou, J.L. and Pichoir, F. (1991) Quantitative Analysis of Homogeneous or Stratified Microvolumes Applying the Model "PAP”. In: Heinrich, K.F.J. and Newbury, D.E., Eds., Electron Probe Quantification, Plenum Press, New York, 31-75. https://doi.org/10.1007/978-1-4899-2617-3 4

[10] Roeder, P.L. and Emslie, R.F. (1970) Olivine-Liquid Equilibrium. Contributions to Mineralogy and Petrology, 29, 275-289. https://doi.org/10.1007/BF00371276

[11] Ngounouno, I. and Déruelle, B. (2007) Petrology of Wehrlites and Clinopyroxenites Xenoliths from Mount Cameroon: Evidence of Mantle Metasomatism. Journal of Cameroon Academy of Science, 7, 35-46.

[12] Morimoto, N. (1989) Nomenclature of Pyroxenes. Canadian Mineralogist, 27, 143-156. https://doi.org/10.2465/minerj.14.198

[13] Wass, S.Y. (1979) Multiple Origins of Clinopyroxenes in Alkali Basaltic Rocks. Lithos, 12, 115-132.

[14] Jagoutz, E., Palme, H., Baddenhausen, H., Blum, K., Cendales, M., Dreibus, G., Spettel, B., Lorenz, V. and Wänke, H. (1979) The Abundances of Major, Minor, and Trace Elements in the Earth's Mantle as Derived from Primitive Ultramafic Nodules. Procedures Lunar Science Conference, 10, 2031-2050.

[15] Nono, A., Déruelle, B., Demaiffe, D. and Kambou, R. (1994) Tchabal Nganha Volcano in Adamawa (Cameroon): Petrology of a Continental Alkaline Lava Series. Journal of Volcanology and Geothermal Research, 60, 147-178.

[16] Caldeira, R. and Munhá, J.M. (2002) Petrology of Ultramafic Xenoliths from São Tomé Island, Cameroon Volcanic Line (Oceanic Sector). Journal of African Earth Science, 34, 231-246.

[17] Lee, D.-C., Halliday, A.N., Davies, G.R., Essene, E.J., Fitton, J.G. and Temdjim, R. (1996) Melt Enrichment of Shallow Depleted Mantle: A Detailed Petrological, Trace Element and Isotopic Study of Mantle-Derived Xenoliths and Megacrysts from the Cameroon Line. Journal of Petrology, 37, 415-441.

https://doi.org/10.1093/petrology/37.2.415

[18] Wandji, P., Tsafack, J.P.F., Bardintzeff, J.M., Nkouathio, D.G., Kagou Dongmo, A., Bellon, H. and Guillou, H. (2009) Xenoliths of Dunites, Wehrlites and Clinopyroxenite in the Basanites from Batoke Volcanic Cone (Mount Cameroon, Central Africa): Petrogenetic Implications. Mineralogy and Petrology, 96, 81-98. https://doi.org/10.1007/s00710-008-0040-3

[19] Ngounouno, I., Dagwaï, N., Kamgang, P. and Deruelle, B. (2008) Petrology of Spinel Lherzolite Xenoliths in Alkali Basalts from Liri, South of the Kapsiki Plateau (Northernmost Cameroon Hot Line). Journal of Cameroon Academy of Science, 8 , 31-42.

[20] Frey, F.A. and Prinz, M. (1978) Ultramafic Inclusions from San Carlos, Arizona: Petrological and Geochemical Data Bearing on Their Petrogenesis. Earth and Planetary Science Letters, 38, 129-176.

[21] Mysen, B.O. and Kushiro, I. (1977) Compositional Variations of Coexisting Phases 
with Degrees of Melting of Peridotite in the Upper Mantle. American Mineralogist, 62, 843-865.

[22] Brey, G.P. and Kohler, T. (1990) Geothermobarometry in Four-Phase Lherzolites II, New Thermobarometers, and Practical Assessment of Existing Their Barometers. Journal of Petrology, 31, 1353-1378. https://doi.org/10.1093/petrology/31.6.1353

[23] Roeder, L.P., Campbell, I.H. and Jameson, H.E. (1979) A Re-Evaluation of the Olivine-Spinel Geothermometer. Contributions to Mineralogy and Petrology, 68, 325-334. https://doi.org/10.1007/BF00371554

[24] Thy, P. (1983) Spinel Minerals in Transitional and Alkali Basaltic Glasses from Iceland. Contributions to Mineralogy and Petrology, 83, 141-149.

https://doi.org/10.1007/BF00373087 